\title{
Forced Oscillation Criteria for a Class of Fractional Partial Differential Equations with Damping Term
}

\author{
Wei Nian Li \\ Department of Mathematics, Binzhou University, Shandong 256603, China \\ Correspondence should be addressed to Wei Nian Li; wnli@263.net
}

Received 2 April 2015; Accepted 27 April 2015

Academic Editor: Kishin Sadarangani

Copyright ( 2015 Wei Nian Li. This is an open access article distributed under the Creative Commons Attribution License, which permits unrestricted use, distribution, and reproduction in any medium, provided the original work is properly cited.

\begin{abstract}
Sufficient conditions are established for the forced oscillation of fractional partial differential equations with damping term of the form $(\partial / \partial t)\left(D_{+, t}^{\alpha} u(x, t)\right)+p(t) D_{+, t}^{\alpha} u(x, t)=a(t) \Delta u(x, t)-q(x, t) u(x, t)+f(x, t),(x, t) \in \Omega \times R_{+} \equiv G$, with one of the two following boundary conditions: $\partial u(x, t) / \partial N=\psi(x, t),(x, t) \in \partial \Omega \times R_{+}$or $u(x, t)=0,(x, t) \in \partial \Omega \times R_{+}$, where $\Omega$ is a bounded domain in $R^{n}$ with a piecewise smooth boundary, $\partial \Omega, R_{+}=[0, \infty), \alpha \in(0,1)$ is a constant, $D_{+, t}^{\alpha} u(x, t)$ is the Riemann-Liouville fractional derivative of order $\alpha$ of $u$ with respect to $t, \Delta$ is the Laplacian in $R^{n}, N$ is the unit exterior normal vector to $\partial \Omega$, and $\psi(x, t)$ is a continuous function on $\partial \Omega \times R_{+}$. The main results are illustrated by some examples.
\end{abstract}

\section{Introduction}

Fractional differential equations are generalizations of classical differential equations to an arbitrary (noninteger) order and have gained increasing attention because of their varied applications in various fields of applied sciences and engineering. In the past few years, the theory of fractional differential equations and their applications have been investigated extensively; for example, see the monographs [1-4].

Recently, the oscillatory behavior of solutions for fractional differential equations was discussed in [5-13] and so forth. In [13], the authors studied the oscillation of solutions to nonlinear fractional differential equations of this form

$$
\begin{aligned}
D_{0+}^{1+\alpha} y(t)+p(t) D_{0+}^{\alpha} y(t)+q(t) f(y(t))=g(t), & t>0,
\end{aligned}
$$

with initial condition $I_{0+}^{1-\alpha} y(0+)=b$, where $b$ is a real number, $\alpha \in(0,1)$ is a constant, and $D_{0+}^{\alpha} y$ is the Riemann-Liouville fractional derivative of order $\alpha$ of $y$.

However, to the best of author's knowledge very little is known regarding the oscillatory behavior of fractional partial differential equations up to now; we refer to [1416]. In particular, nothing is known regarding the oscillation properties of the problems (1)-(2) and (1)-(3) up to now.
In this paper we investigate the forced oscillation of fractional partial differential equations with damping term of the form

$$
\begin{aligned}
\frac{\partial}{\partial t} & \left(D_{+, t}^{\alpha} u(x, t)\right)+p(t) D_{+, t}^{\alpha} u(x, t) \\
& =a(t) \Delta u(x, t)-q(x, t) u(x, t)+f(x, t), \\
& (x, t) \in \Omega \times R_{+} \equiv G,
\end{aligned}
$$

where $\Omega$ is a bounded domain in $R^{n}$ with a piecewise smooth boundary $\partial \Omega, R_{+}=[0, \infty), \alpha \in(0,1)$ is a constant, $D_{+, t}^{\alpha} u(x, t)$ is the Riemann-Liouville fractional derivative of order $\alpha$ of $u$ with respect to $t$, and $\Delta$ is the Laplacian in $R^{n}$.

We assume throughout this paper that

(A1) $a \in C\left(R_{+} ;(0, \infty)\right), p \in C\left(R_{+} ; R\right)$;

(A2) $q \in C\left(\bar{G} ; R_{+}\right)$, and $q(t)=\min _{x \in \bar{\Omega}} q(x, t)$;

(A3) $f \in C(\bar{G} ; R)$.

Consider one of the two following boundary conditions:

$$
\frac{\partial u(x, t)}{\partial N}=\psi(x, t), \quad(x, t) \in \partial \Omega \times R_{+},
$$

or

$$
u(x, t)=0, \quad(x, t) \in \partial \Omega \times R_{+},
$$


where $N$ is the unit exterior normal vector to $\partial \Omega$ and $\psi(x, t)$ is a continuous function on $\partial \Omega \times R_{+}$.

By a solution of the problems (1)-(2) (or (1)-(3)), we mean a function $u(x, t)$ which satisfies (1) on $\bar{G}$ and the boundary condition (2) (or (3)).

A solution $u(x, t)$ of the problems (1)-(2) (or (1)-(3)) is said to be oscillatory in $G$ if it is neither eventually positive nor eventually negative, otherwise it is nonoscillatory.

Definition 1. The Riemann-Liouville fractional partial derivative of order $0<\alpha<1$ with respect to $t$ of a function $u(x, t)$ is given by

$$
D_{+, t}^{\alpha} u(x, t):=\frac{1}{\Gamma(1-\alpha)} \frac{\partial}{\partial t} \int_{0}^{t}(t-\xi)^{-\alpha} u(x, \xi) d \xi,
$$

provided the right hand side is pointwise defined on $R_{+}$, where $\Gamma$ is the gamma function.

Definition 2. The Riemann-Liouville fractional integral of order $\alpha>0$ of a function $y: R_{+} \rightarrow R$ on the half-axis $R_{+}$is given by

$$
I_{+}^{\alpha} y(t):=\frac{1}{\Gamma(\alpha)} \int_{0}^{t}(t-\xi)^{\alpha-1} y(\xi) d \xi \quad \text { for } t>0,
$$

provided the right hand side is pointwise defined on $R_{+}$.

Definition 3. The Riemann-Liouville fractional derivative of order $\alpha>0$ of a function $y: R_{+} \rightarrow R$ on the half-axis $R_{+}$is given by

$$
\begin{aligned}
D_{+}^{\alpha} y(t) & :=\frac{d^{\lceil\alpha\rceil}}{d t^{\lceil\alpha\rceil}}\left(I_{+}^{\lceil\alpha\rceil-\alpha} y\right)(t) \\
& =\frac{1}{\Gamma(\lceil\alpha\rceil-\alpha)} \frac{d^{\lceil\alpha\rceil}}{d t^{\lceil\alpha\rceil}} \int_{0}^{t}(t-\xi)^{\lceil\alpha\rceil-\alpha-1} y(\xi) d \xi \\
& \text { for } t>0,
\end{aligned}
$$

provided the right hand side is pointwise defined on $R_{+}$, where $\lceil\alpha\rceil$ is the ceiling function of $\alpha$.

\section{Main Results}

In this section, we establish the oscillation of the problems (1)-(2) and (1)-(3). We firstly introduce the following lemmas which are very useful in the proof of our main results.

Lemma 4 (see [17]). The smallest eigenvalue $\beta_{0}$ of the Dirichlet problem,

$$
\begin{aligned}
\Delta \omega(x)+\beta \omega(x) & =0, \quad \text { in } \Omega, \\
\omega(x) & =0, \quad \text { on } \partial \Omega,
\end{aligned}
$$

is positive and the corresponding eigenfunction $\varphi(x)$ is positive in $\Omega$.

Lemma 5 (see [2, page 74]). Let $\alpha \geq 0, m \in N$, and $D=d / d x$. If the fractional derivatives $D_{+}^{\alpha} y(t)$ and $D_{+}^{\alpha+m} y(t)$ exist, then

$$
D^{m}\left(D_{+}^{\alpha} y(t)\right)=D_{+}^{\alpha+m} y(t) .
$$

Lemma 6 (see [2, page 75]). Let $\alpha \in(0,1)$ and $I_{+}^{1-\alpha} y(t)$ be the fractional integral (5) of order $1-\alpha$, then

$$
I_{+}^{\alpha}\left(D_{+}^{\alpha} y(t)\right)=y(t)-\frac{I_{+}^{1-\alpha} y(0)}{\Gamma(\alpha)} t^{\alpha-1} .
$$

For convenience, one uses the following notations in this paper:

$$
\begin{aligned}
& U(t)=\int_{\Omega} u(x, t) d x, \\
& \Psi(t)=\int_{\partial \Omega} \psi(x, t) d S, \\
& F(t)=\int_{\Omega} f(x, t) d x, \\
& H(t)=F(t)+a(t) \Psi(t), \\
& W(t)=\int_{\Omega} u(x, t) \varphi(x) d x, \\
& Y(t)=\int_{\Omega} f(x, t) \varphi(x) d x, \\
& t \geq 0,
\end{aligned}
$$

where $d S$ is the surface element on $\partial \Omega$.

Theorem 7. Assume that

$$
\lim _{t \rightarrow 0} I_{+}^{1-\alpha} U(t)=C_{0},
$$

where $C_{0}$ is a constant. If

$$
\begin{aligned}
& \liminf _{t \rightarrow \infty} \int_{0}^{t} \frac{(t-\xi)^{\alpha-1}}{V(\xi)}\left(C+\int_{t_{0}}^{\xi} H(s) V(s) d s\right) d \xi<0, \\
& \limsup _{t \rightarrow \infty} \int_{0}^{t} \frac{(t-\xi)^{\alpha-1}}{V(\xi)}\left(C+\int_{t_{0}}^{\xi} H(s) V(s) d s\right) d \xi \\
& \quad>0
\end{aligned}
$$

then every solution $u(x, t)$ of the problems (1)-(2) is oscillatory in $G$, where $V(t)=\exp \int_{t_{0}}^{t} p(\xi) d \xi$ and $C$ is a constant.

Proof. Suppose to the contrary that there is a nonoscillatory solution $u(x, t)$ of the problems (1)-(2) which has no zero in $\Omega \times\left[t_{0}, \infty\right)$ for some $t_{0} \geq 0$. Then $u(x, t)>0$ or $u(x, t)<0$ for $t \geq t_{0}$.

Case $1\left(u(x, t)>0, t \geq t_{0}\right)$. Integrating (1) with respect to $x$ over the domain $\Omega$, we have

$$
\begin{aligned}
& \frac{d}{d t}\left(\int_{\Omega} D_{+, t}^{\alpha} u(x, t) d x\right)+p(t) \int_{\Omega} D_{+, t}^{\alpha} u(x, t) d x \\
& =a(t) \int_{\Omega} \Delta u(x, t) d x-\int_{\Omega} q(x, t) u(x, t) d x \\
& \quad+\int_{\Omega} f(x, t) d x, \quad t \geq t_{0} .
\end{aligned}
$$


Green's formula and (2) yield

$$
\begin{aligned}
\int_{\Omega} \Delta u(x, t) d x & =\int_{\partial \Omega} \frac{\partial u(x, t)}{\partial N} d S=\int_{\partial \Omega} \psi(x, t) d S \\
& =\Psi(t), \quad t \geq t_{0} .
\end{aligned}
$$

From (A2), it is easy to see that

$$
\int_{\Omega} q(x, t) u(x, t) d x \geq q(t) \int_{\Omega} u(x, t) d x, \quad t \geq t_{0} .
$$

By Lemma 5, it follows from (13)-(15) that

$$
D_{+}^{1+\alpha} U(t)+p(t) D_{+}^{\alpha} U(t) \leq-q(t) U(t)+H(t),
$$

$$
t \geq t_{0} \text {. }
$$

From (16), we easily see that

$$
\begin{aligned}
\left(\left(D_{+}^{\alpha} U(t)\right) V(t)\right)^{\prime}= & \left(D_{+}^{1+\alpha} U(t)\right) V(t) \\
& +\left(D_{+}^{\alpha} U(t)\right) p(t) V(t) \\
\leq & -q(t) U(t) V(t)+H(t) V(t) \\
\leq & H(t) V(t), \quad t \geq t_{0} .
\end{aligned}
$$

Integrating both sides of the above inequality from $t_{0}$ to $t$, we obtain

$$
\begin{aligned}
\left(D_{+}^{\alpha} U(t)\right) V(t) \leq & \left(D_{+}^{\alpha} U\left(t_{0}\right)\right) V\left(t_{0}\right) \\
& +\int_{t_{0}}^{t} H(s) V(s) d s \\
= & C+\int_{t_{0}}^{t} H(s) V(s) d s,
\end{aligned}
$$

where $C=\left(D_{+}^{\alpha} U\left(t_{0}\right)\right) V\left(t_{0}\right)$. Using Lemma 6, it follows from (18) that

$$
\begin{aligned}
& U(t) \\
& \leq \frac{I_{+}^{1-\alpha} U(0)}{\Gamma(\alpha)} t^{\alpha-1} \\
& \quad+I_{+}^{\alpha}\left(\frac{C}{V(t)}+\frac{1}{V(t)} \int_{t_{0}}^{t} H(s) V(s) d s\right) \\
& =\frac{C_{0}}{\Gamma(\alpha)} t^{\alpha-1} \\
& \quad+\frac{1}{\Gamma(\alpha)} \int_{0}^{t} \frac{(t-\xi)^{\alpha-1}}{V(\xi)}\left(C+\int_{t_{0}}^{\xi} H(s) V(s) d s\right) d \xi .
\end{aligned}
$$

Taking $t \rightarrow \infty$ in (19), we have

$$
\begin{gathered}
\liminf _{t \rightarrow \infty} U(t) \leq \limsup _{t \rightarrow \infty} \frac{C_{0}}{\Gamma(\alpha)} t^{\alpha-1}+\liminf _{t \rightarrow \infty} \frac{1}{\Gamma(\alpha)} \\
\cdot \int_{0}^{t} \frac{(t-\xi)^{\alpha-1}}{V(\xi)}\left(C+\int_{t_{0}}^{\xi} H(s) V(s) d s\right) d \xi<0,
\end{gathered}
$$

which contradicts the fact that $U(t)>0$.
Case $2\left(u(x, t)<0, t \geq t_{0}\right)$. Using the procedure of the proof of Case 1, we conclude that (13) and (14) are satisfied. From (A2), we have

$$
\int_{\Omega} q(x, t) u(x, t) d x \leq q(t) \int_{\Omega} u(x, t) d x, \quad t \geq t_{0} .
$$

Combining (13), (14), and (21), we have

$$
D_{+}^{1+\alpha} U(t)+p(t) D_{+}^{\alpha} U(t) \geq-q(t) U(t)+H(t),
$$

$$
t \geq t_{0} \text {. }
$$

It follows from (22) that

$$
\begin{aligned}
\left(\left(D_{+}^{\alpha} U(t)\right) V(t)\right)^{\prime}= & \left(D_{+}^{1+\alpha} U(t)\right) V(t) \\
& +\left(D_{+}^{\alpha} U(t)\right) p(t) V(t) \\
\geq & -q(t) U(t) V(t)+H(t) V(t) \\
\geq & H(t) V(t), \quad t \geq t_{0} .
\end{aligned}
$$

Integrating both sides of the above inequality from $t_{0}$ to $t$, we obtain

$$
\begin{aligned}
\left(D_{+}^{\alpha} U(t)\right) V(t) \geq & \left(D_{+}^{\alpha} U\left(t_{0}\right)\right) V\left(t_{0}\right) \\
& +\int_{t_{0}}^{t} H(s) V(s) d s \\
= & C+\int_{t_{0}}^{t} H(s) V(s) d s .
\end{aligned}
$$

Using Lemma 6, from (24) we obtain

$U(t)$

$$
\begin{aligned}
\geq & \frac{I_{+}^{1-\alpha} U(0)}{\Gamma(\alpha)} t^{\alpha-1} \\
& +I_{+}^{\alpha}\left(\frac{C}{V(t)}+\frac{1}{V(t)} \int_{t_{0}}^{t} H(s) V(s) d s\right) \\
= & \frac{C_{0}}{\Gamma(\alpha)} t^{\alpha-1} \\
& +\frac{1}{\Gamma(\alpha)} \int_{0}^{t} \frac{(t-\xi)^{\alpha-1}}{V(\xi)}\left(C+\int_{t_{0}}^{\xi} H(s) V(s) d s\right) d \xi .
\end{aligned}
$$

Taking $t \rightarrow \infty$ in (25), we have

$$
\begin{gathered}
\limsup _{t \rightarrow \infty} U(t) \geq \liminf _{t \rightarrow \infty} \frac{C_{0}}{\Gamma(\alpha)} t^{\alpha-1}+\limsup _{t \rightarrow \infty} \frac{1}{\Gamma(\alpha)} \\
\cdot \int_{0}^{t} \frac{(t-\xi)^{\alpha-1}}{V(\xi)}\left(C+\int_{t_{0}}^{\xi} H(s) V(s) d s\right) d \xi>0
\end{gathered}
$$

which contradicts the fact that $U(t)<0$. This completes the proof of Theorem 7 .

Theorem 8. Assume that

$$
\lim _{t \rightarrow 0} I_{+}^{1-\alpha} W(t)=C_{1},
$$


where $C_{1}$ is a constant. If

$$
\begin{aligned}
& \liminf _{t \rightarrow \infty} \int_{0}^{t} \frac{(t-\xi)^{\alpha-1}}{V(\xi)}\left(C+\int_{t_{0}}^{\xi} Y(s) V(s) d s\right) d \xi<0, \\
& \limsup _{t \rightarrow \infty} \int_{0}^{t} \frac{(t-\xi)^{\alpha-1}}{V(\xi)}\left(C+\int_{t_{0}}^{\xi} Y(s) V(s) d s\right) d \xi>0,
\end{aligned}
$$

then every solution $u(x, t)$ of the problems (1)-(3) is oscillatory in $G$, where $V(t)=\exp \int_{t_{0}}^{t} p(\xi) d \xi$ and $C$ is a constant.

Proof. Suppose to the contrary that there is a nonoscillatory solution $u(x, t)$ of the problems (1)-(3) which has no zero in $\Omega \times\left[t_{0}, \infty\right)$ for some $t_{0} \geq 0$. Then $u(x, t)>0$ or $u(x, t)<0$ for $t \geq t_{0}$.

Case $1\left(u(x, t)>0, t \geq t_{0}\right)$. Multiplying both sides of (1) by $\varphi(x)$ and integrating with respect to $x$ over the domain $\Omega$, we have

$$
\begin{aligned}
& \frac{d}{d t}\left(\int_{\Omega} D_{+, t}^{\alpha} u(x, t) \varphi(x) d x\right) \\
& \quad+p(t) \int_{\Omega} D_{+, t}^{\alpha} u(x, t) \varphi(x) d x \\
& =a(t) \int_{\Omega} \Delta u(x, t) \varphi(x) d x \\
& \quad-\int_{\Omega} q(x, t) u(x, t) \varphi(x) d x \\
& \quad+\int_{\Omega} f(x, t) \varphi(x) d x, \quad t \geq t_{0} .
\end{aligned}
$$

Green's formula and (3) yield

$$
\begin{aligned}
\int_{\Omega} \Delta u(x, t) \varphi(x) d x & =\int_{\Omega} u(x, t) \Delta \varphi(x) d x \\
& =-\beta_{0} \int_{\Omega} u(x, t) \varphi(x) d x
\end{aligned}
$$

$$
t \geq t_{0}
$$

From (A2), it is easy to see that

$$
\begin{array}{r}
\int_{\Omega} q(x, t) u(x, t) \varphi(x) d x \geq q(t) \int_{\Omega} u(x, t) \varphi(x) d x \\
t \geq t_{0}
\end{array}
$$

Combining (29)-(31), we easily see that

$$
\begin{aligned}
& D_{+}^{1+\alpha} W(t)+p(t) D_{+}^{\alpha} W(t) \\
& \quad \leq-\left(\beta_{0} a(t)+q(t)\right) W(t)+Y(t), \quad t \geq t_{0} .
\end{aligned}
$$

The remainder of the proof is similar to that of Case 1 of Theorem 7 and we can obtain a contradiction to $W(t)>0$.

Case $2\left(u(x, t)<0, t \geq t_{0}\right)$. Using the procedure of the proof of Case 1, we conclude that (29) and (30) are satisfied. From (A2), we have

$$
\begin{array}{r}
\int_{\Omega} q(x, t) u(x, t) \varphi(x) d x \leq q(t) \int_{\Omega} u(x, t) \varphi(x) d x \\
t \geq t_{0} .
\end{array}
$$

Combining (29), (30), and (33), we obtain

$$
\begin{aligned}
& D_{+}^{1+\alpha} W(t)+p(t) D_{+}^{\alpha} W(t) \\
& \quad \geq-\left(\beta_{0} a(t)+q(t)\right) W(t)+Y(t), \quad t \geq t_{0} .
\end{aligned}
$$

Using a similar way in the proof of Case 2 of Theorem 7 , we can obtain a contradiction to $W(t)<0$. The proof of Theorem 8 is complete.

\section{Examples}

Example 1. Consider the following fractional partial differential equation:

$$
\begin{array}{r}
\frac{\partial}{\partial t}\left(D_{+, t}^{1 / 2} u(x, t)\right)-D_{+, t}^{1 / 2} u(x, t) \\
=\frac{1}{\pi} \Delta u(x, t)-\left(x^{2}+t^{2}\right) u(x, t)+e^{t} \sin t \sin x, \\
\quad(x, t) \in(0, \pi) \times R_{+},
\end{array}
$$

with the boundary condition

$$
-\frac{\partial u(0, t)}{\partial x}=\frac{\partial u(\pi, t)}{\partial x}=-e^{t} \sin t, \quad t \geq 0 .
$$

Here $\alpha=1 / 2, \Omega=(0, \pi), n=1, p(t)=-1, a(t)=1 / \pi$, $q(x, t)=x^{2}+t^{2}$, and $f(x, t)=e^{t} \sin t \sin x$. It is obvious that $V(t)=e^{t_{0}-t}, q(t)=t^{2}, \Psi(t)=-\pi e^{t} \sin t$, and

$$
\begin{aligned}
F(t) & =\int_{\Omega} f(x, t) d x=\int_{0}^{\pi} e^{t} \sin t \sin x d x \\
& =2 e^{t} \sin t, \\
H(t) & =F(t)+a(t) \Psi(t)=e^{t} \sin t .
\end{aligned}
$$

Hence

$$
\begin{aligned}
\int_{t_{0}}^{\xi} H(s) V(s) d s & =\int_{t_{0}}^{\xi} e^{s} \sin s e^{t_{0}-s} d s \\
& =e^{t_{0}}\left(-\cos \xi+\cos t_{0}\right) .
\end{aligned}
$$


Letting $t_{0}=\pi / 2$, we obtain

$$
\begin{aligned}
& \int_{0}^{t} \frac{(t-\xi)^{-1 / 2}}{V(\xi)}\left(C+\int_{t_{0}}^{\xi} H(s) V(s) d s\right) d \xi \\
& \quad=\int_{0}^{t}(t-\xi)^{-1 / 2} e^{\xi-\pi / 2}\left(C+\int_{\pi / 2}^{\xi} H(s) V(s) d s\right) d \xi \\
& \quad=\int_{0}^{t}(t-\xi)^{-1 / 2} e^{\xi-\pi / 2}\left(C-e^{\pi / 2} \cos \xi\right) d \xi
\end{aligned}
$$

Setting $t-\xi=s^{2}$, above integral (39) can be written as the following form:

$$
\begin{aligned}
\int_{\sqrt{t}}^{0} & \frac{1}{s}\left(C e^{t-s^{2}-\pi / 2}-e^{t-s^{2}} \cos \left(t-s^{2}\right)\right)(-2 s) d s \\
& =2 C e^{t-\pi / 2} \int_{0}^{\sqrt{t}} e^{-s^{2}} d s \\
& -2 e^{t} \int_{0}^{\sqrt{t}} e^{-s^{2}} \cos \left(t-s^{2}\right) d s \\
& =2 C e^{t-\pi / 2} \int_{0}^{\sqrt{t}} e^{-s^{2}} d s-2 e^{t} \cos t \int_{0}^{\sqrt{t}} e^{-s^{2}} \cos s^{2} d s \\
& -2 e^{t} \sin t \int_{0}^{\sqrt{t}} e^{-s^{2}} \sin s^{2} d s \\
& =2 e^{t}\left[C e^{-\pi / 2} \int_{0}^{\sqrt{t}} e^{-s^{2}} d s-\cos t \int_{0}^{\sqrt{t}} e^{-s^{2}} \cos s^{2} d s\right. \\
& \left.-\sin t \int_{0}^{\sqrt{t}} e^{-s^{2}} \sin s^{2} d s\right] .
\end{aligned}
$$

Noting that

$$
\begin{array}{r}
\left|e^{-s^{2}} \cos s^{2}\right| \leq e^{-s^{2}}, \\
\left|e^{-s^{2}} \sin s^{2}\right| \leq e^{-s^{2}}, \\
\lim _{t \rightarrow \infty} \int_{0}^{\sqrt{t}} e^{-s^{2}} d s=\frac{\sqrt{\pi}}{2},
\end{array}
$$

we obtain that

$$
\begin{aligned}
& \lim _{t \rightarrow \infty} \int_{0}^{\sqrt{t}} e^{-s^{2}} \cos s^{2} d s \\
& \lim _{t \rightarrow \infty} \int_{0}^{\sqrt{t}} e^{-s^{2}} \sin s^{2} d s
\end{aligned}
$$

are convergent.
Therefore, combining (39)-(41) and noting the fact that (42) are convergent, by careful calculation, we can get

$$
\begin{aligned}
& \liminf _{t \rightarrow \infty} \int_{0}^{t} \frac{(t-\xi)^{\alpha-1}}{V(\xi)}\left(C+\int_{t_{0}}^{\xi} H(s) V(s) d s\right) d \xi \\
& \quad \leq-\infty<0, \\
& \limsup _{t \rightarrow \infty} \int_{0}^{t} \frac{(t-\xi)^{\alpha-1}}{V(\xi)}\left(C+\int_{t_{0}}^{\xi} H(s) V(s) d s\right) d \xi \\
& \geq+\infty>0,
\end{aligned}
$$

which shows that all the conditions of Theorem 7 are fulfilled. Then every solution of the problems (35)-(36) oscillates in $(0, \pi) \times R_{+}$.

Example 2. Consider the following fractional partial differential equation:

$$
\begin{aligned}
\frac{\partial}{\partial t}\left(D_{+, t}^{1 / 2} u(x, t)\right)-D_{+, t}^{1 / 2} u(x, t) \\
=e^{-t} \Delta u(x, t)-\left(2 x^{2}+t^{2}\right) u(x, t) \\
\quad+e^{t-\pi} \sin t \sin x, \quad(x, t) \in(0, \pi) \times R_{+},
\end{aligned}
$$

with the boundary condition

$$
u(0, t)=u(\pi, t)=0, \quad t \geq 0 .
$$

Here $\alpha=1 / 2, \Omega=(0, \pi), n=1, p(t)=-1, a(t)=e^{-t}$, $q(x, t)=2 x^{2}+t^{2}$, and $f(x, t)=e^{t-\pi} \sin t \sin x$.

It is obvious that $V(t)=e^{t_{0}-t}, q(t)=t^{2}, \beta_{0}=1$, and $\varphi(x)=\sin x$. Therefore,

$$
\begin{aligned}
Y(t) & =\int_{\Omega} f(x, t) \varphi(x) d x=\int_{0}^{\pi} e^{t-\pi} \sin t \sin ^{2} x d x \\
& =\frac{\pi e^{-\pi}}{2} e^{t} \sin t .
\end{aligned}
$$

Hence

$$
\begin{aligned}
\int_{t_{0}}^{\xi} Y(s) V(s) d s & =\frac{\pi e^{-\pi}}{2} \int_{t_{0}}^{\xi} e^{s} \sin s e^{t_{0}-s} d s \\
& =\frac{\pi e^{t_{0}-\pi}}{2}\left(-\cos \xi+\cos t_{0}\right) .
\end{aligned}
$$

Using a similar way in Example 1, we can obtain

$$
\begin{aligned}
& \liminf _{t \rightarrow \infty} \int_{0}^{t} \frac{(t-\xi)^{\alpha-1}}{V(\xi)}\left(C+\int_{t_{0}}^{\xi} Y(s) V(s) d s\right) d \xi \\
& \quad \leq-\infty<0 \\
& \limsup _{t \rightarrow \infty} \int_{0}^{t} \frac{(t-\xi)^{\alpha-1}}{V(\xi)}\left(C+\int_{t_{0}}^{\xi} Y(s) V(s) d s\right) d \xi \\
& \quad \geq+\infty>0 .
\end{aligned}
$$

Therefore, by Theorem 8 , it is easy to see that every solution of the problems (44)-(45) is oscillatory in $(0, \pi) \times R_{+}$. 


\section{Conflict of Interests}

The author declares that there is no conflict of interests regarding the publication of this paper.

\section{Acknowledgments}

This work is supported by the National Natural Science Foundation of China (10971018). The author thanks the referee for his valuable comments and suggestions on this paper.

\section{References}

[1] I. Podlubny, Fractional Differential Equations, vol. 198, Academic Press, San Diego, Calif, USA, 1999.

[2] A. A. Kilbas, H. M. Srivastava, and J. J. Trujillo, Theory and Applications of Fractional Differential Equations, Elsevier Science B.V, Amsterdam, The Netherlands, 2006.

[3] S. Das, Functional Fractional Calculus for System Identification and Controls, Springer, Berlin, Germany, 2008.

[4] Y.Zhou, Basic Theory of Fractional Differential Equations, World Scientific, Singapore, 2014.

[5] S. Öğrekçi, "Interval oscillation criteria for functional differential equations of fractional order," Advances in Difference Equations, vol. 2015, article 3, 2015.

[6] Y. Bolat, "On the oscillation of fractional-order delay differential equations with constant coefficients," Communications in Nonlinear Science and Numerical Simulation, vol. 19, no. 11, pp. 39883993, 2014.

[7] D.-X. Chen, "Oscillation criteria of fractional differential equations," Advances in Difference Equations, vol. 2012, article 33, 2012.

[8] S. R. Grace, R. P. Agarwal, P. J. Y. Wong, and A. Zafer, "On the oscillation of fractional differential equations," Fractional Calculus and Applied Analysis, vol. 15, no. 2, pp. 222-231, 2012.

[9] B. Zheng, "Oscillation for a class of nonlinear fractional differential equations with damping term," Journal of Advanced Mathematical Studies, vol. 6, no. 1, pp. 107-115, 2013.

[10] Z. Han, Y. Zhao, Y. Sun, and C. Zhang, "Oscillation for a class of fractional differential equation," Discrete Dynamics in Nature and Society, vol. 2013, Article ID 390282, 6 pages, 2013.

[11] C. Qi and J. Cheng, "Interval oscillation criteria for a class of fractional differential equations with damping term," Mathematical Problems in Engineering, vol. 2013, Article ID 301085, 8 pages, 2013.

[12] D.-X. Chen, P.-X. Qu, and Y.-H. Lan, "Forced oscillation of certain fractional differential equations," Advances in Difference Equations, vol. 2013, article 125, 10 pages, 2013.

[13] J. Yang, A. Liu, and T. Liu, "Forced oscillation of nonlinear fractional differential equations with damping term," Advances in Difference Equations, vol. 2015, 7 pages, 2015.

[14] P. Prakash, S. Harikrishnan, J. J. Nieto, and J.-H. Kim, "Oscillation of a time fractional partial differential equation," Electronic Journal of Qualitative Theory of Differential Equations, vol. 15, pp. 1-10, 2014.

[15] S. Harikrishnan, P. Prakash, and J. J. Nieto, "Forced oscillation of solutions of a nonlinear fractional partial differential equation," Applied Mathematics and Computation, vol. 254, pp. 14-19, 2015.

[16] P. Prakash, S. Harikrishnan, and M. Benchohra, "Oscillation of certain nonlinear fractional partial differential equation with damping term," Applied Mathematics Letters, vol. 43, pp. 72-79, 2015.

[17] R. Courant and D. Hilbert, Methods of Mathematical Physics, vol. 1, Interscience, New York, NY, USA, 1966. 


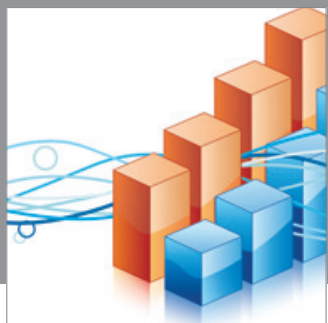

Advances in

Operations Research

mansans

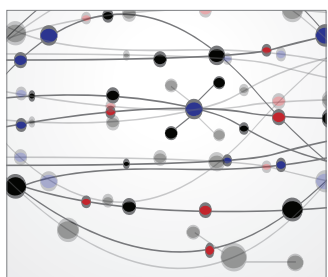

The Scientific World Journal
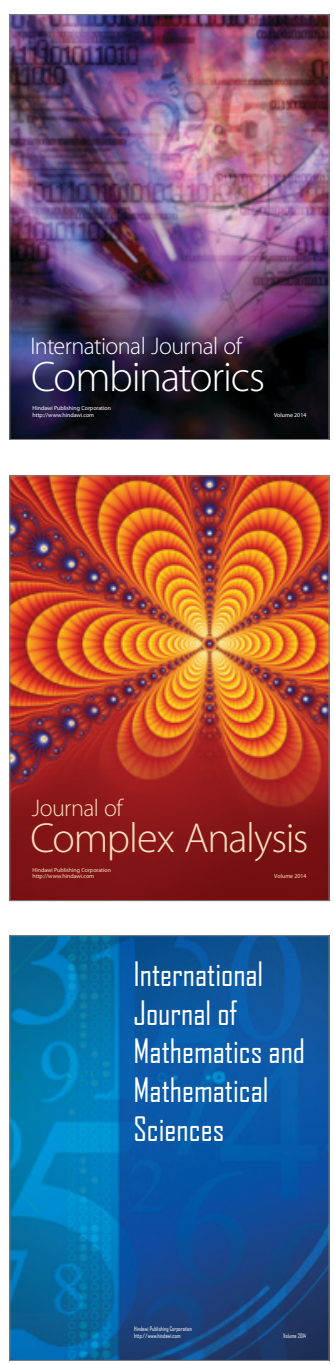
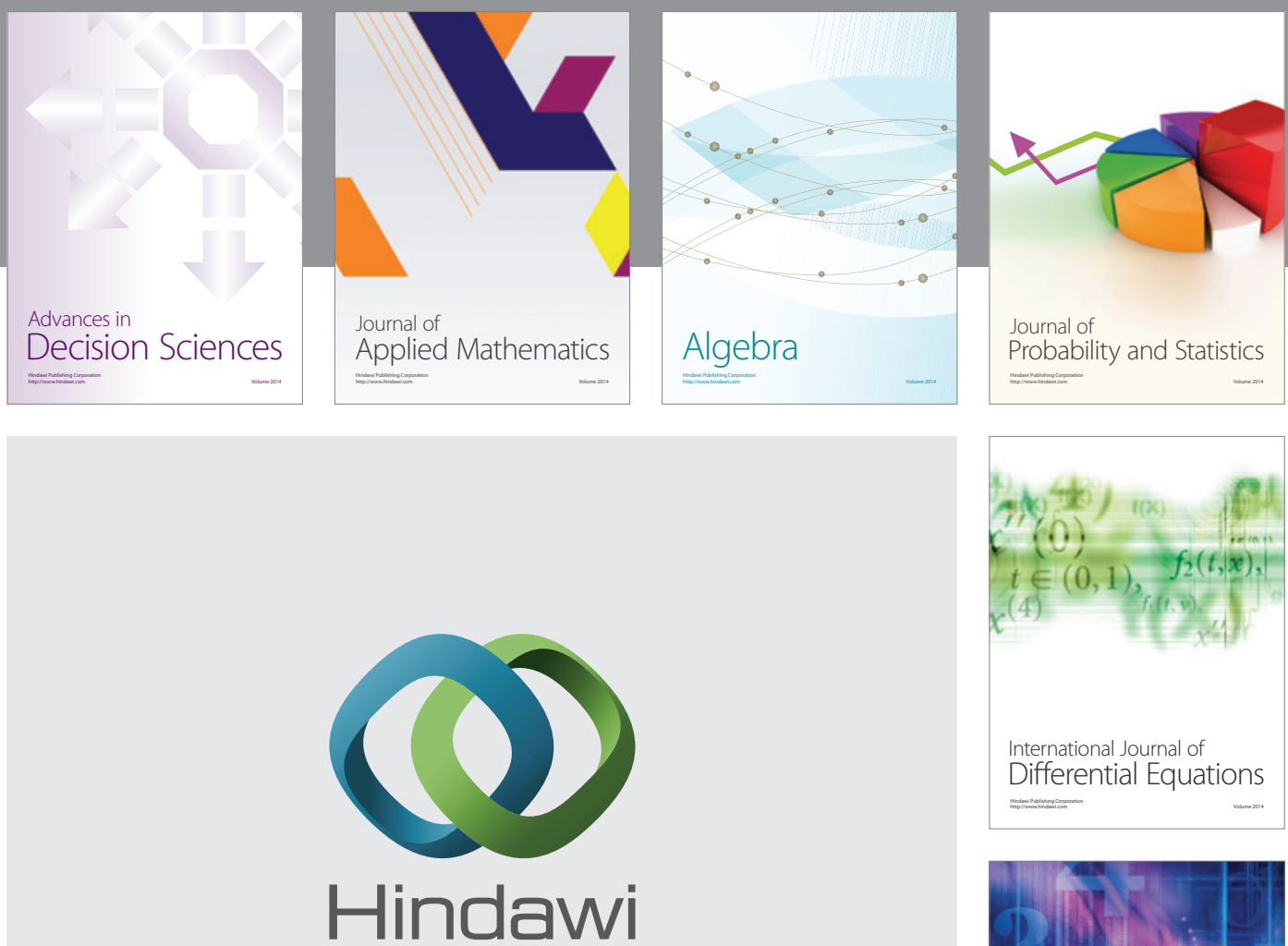

Submit your manuscripts at http://www.hindawi.com
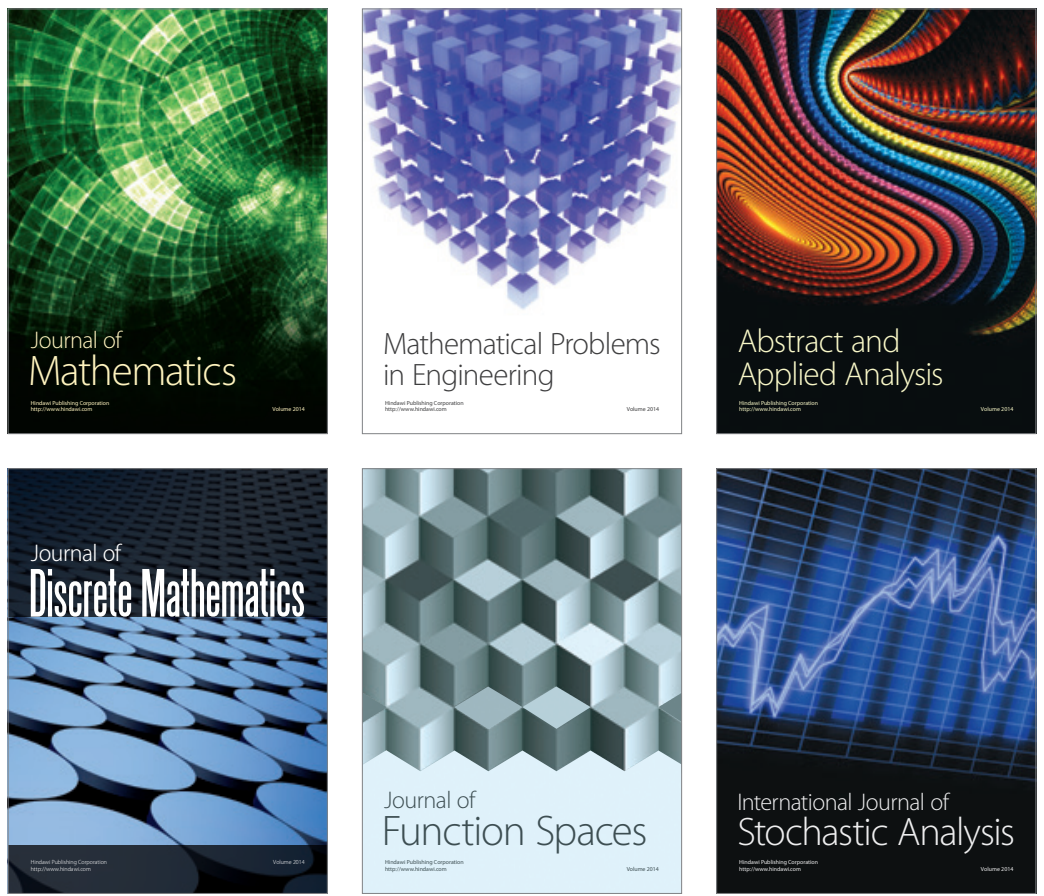

Journal of

Function Spaces

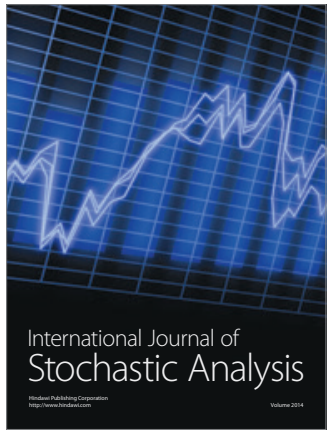

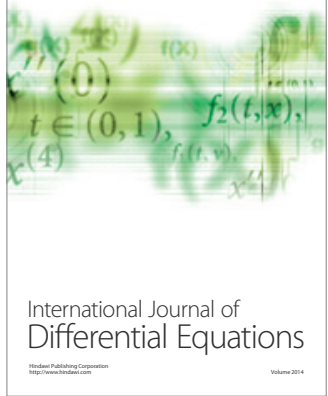
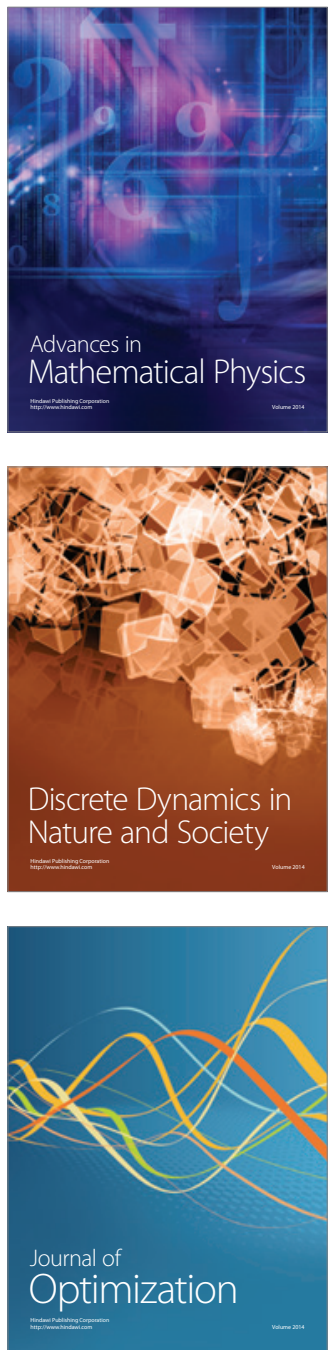